\title{
Catheter ablation of a monofocal premature ventricular complex triggering idiopathic ventricular fibrillation
}

\author{
S Takatsuki, H Mitamura, S Ogawa
}

\begin{abstract}
A 62 year old man was admitted for evaluation of recurrent episodes of syncope. A surface ECG showed frequent repetitive premature ventricular complexes of right ventricular outflow tract origin. Ventricular fibrillation was inducible by programmed electrical stimulation but otherwise cardiac evaluation was unremarkable. A diagnosis of idiopathic ventricular fibrillation was made and an implantable cardioverter-defibrillator (ICD) was installed. However, spontaneous ventricular fibrillation recurred, requiring repeated ICD discharges. The ventricular fibrillation was reproducibly triggered by a single premature ventricular complex with a specific QRS morphology. Radiofrequency catheter ablation was carried out to eradicate this complex. No ventricular fibrillation has developed after this procedure, and the patient does not require drug treatment.

(Heart 2001;86:e3)
\end{abstract}

Keywords: ventricular fibrillation; radiofrequency ablation

A 62 year old man was admitted to Keio University Hospital for evaluation and treatment of syncope. Thirteen years previously he began to feel skipping of heart beats during exertion. Three months later he experienced syncope,

Accepted 7 March 2001

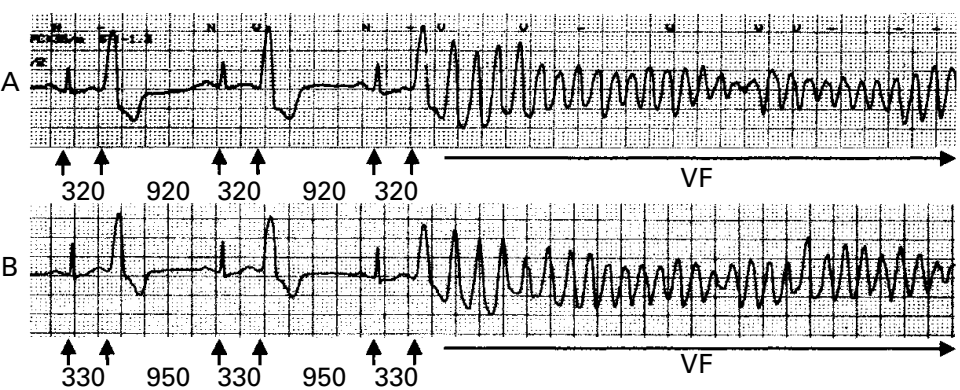

Figure 1 Reproducible pattern of spontaneous initiation of ventricular fibrillation (VF). (A) Spontaneous VF documented on the monitored ECG four days after implantable cardioverter-defibrillator (ICD) installation. The coupling interval of the first premature ventricular complex triggering $V F$ was $320 \mathrm{~ms}$, which was $i d e n t i c a l$ to that of the preceding bigeminal isolated premature ventricular complex. (B) Spontaneous VF recurrence was recorded by the monitored ECG six days after ICD implantation. The manner of induction was almost the same as in $(A)$, with a bigeminal premature ventricular complex (coupling interval $330 \mathrm{~ms}$ ) triggering $V F$. for which he was admitted to hospital for the first time. No structural heart disease was detected by physical, echocardiographic, scintigraphic, or angiographic investigations. A 12 lead ECG showed no abnormality, and in particular there was no abnormal ST elevation or QT prolongation. However, ECG monitoring disclosed frequent premature ventricular complexes of right ventricular outflow tract origin with occasional runs. The patient then underwent electrophysiological studies during which ventricular fibrillation was induced by double extrastimuli at the right ventricular apex. A diagnosis of idiopathic ventricular fibrillation was made and he was prescribed mexiletine $400 \mathrm{mg} /$ day and atenolol $50 \mathrm{mg} /$ day to suppress the complexes responsible for the syncope, which was considered potentially fatal.

His subsequent course was uneventful except for occasional premature ventricular complexes and non-sustained ventricular tachycardias until three days before the most recent admission, when he had a second episode of syncope. While apparently asleep, he suddenly fell to the floor from the bed, with continued loss of consciousness and urinary incontinence. After admission to hospital, an implantable cardioverter-defibrillator (ICD) was installed because the syncope was considered to have been the result of a ventricular tachyarrhythmia. A surface ECG showed frequent premature ventricular complexes from the right ventricular outflow tract, often with bigeminal cycles, and 24 hour ECG monitoring documented 37000 beats a day of monofocal premature ventricular complexes.

Four days after the implantation of the ICD, and while he was in the bed on continuous ECG monitoring, ventricular fibrillation developed suddenly, triggered by a premature ventricular complex of the same morphology as those seen daily during the ECG monitoring (fig 1A). This episode of ventricular fibrillation was successfully treated by an internal $30 \mathrm{~J}$ shock. Two days later, he had two additional episodes of spontaneous ventricular fibrillation, the initiation pattern being exactly the same as that of the earlier event-characterised by a premature ventricular complex of the same QRS morphology appearing as a bigeminal cycle followed suddenly by the induction of ventricular fibrillation (fig 1B). Intravenous 


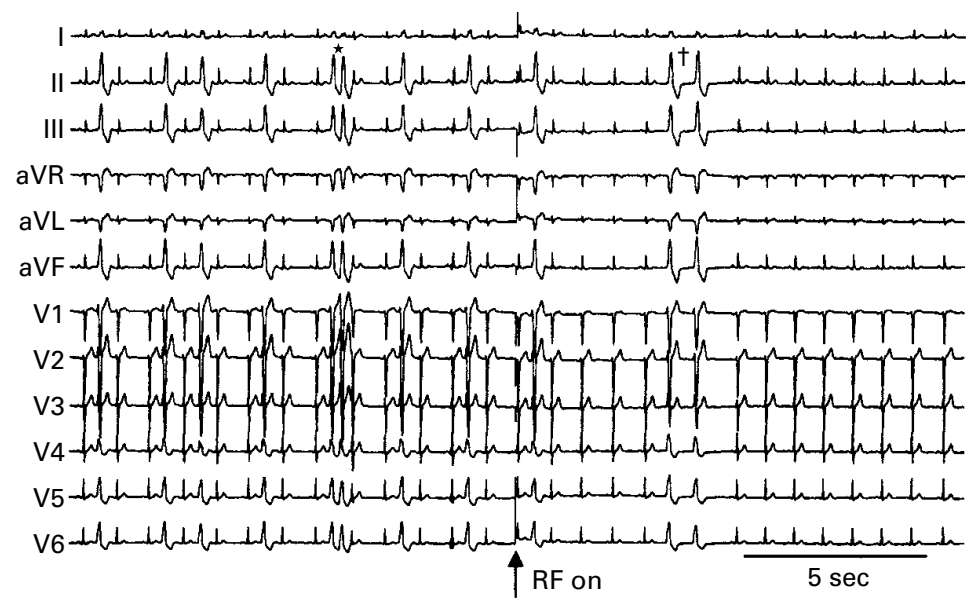

Figure 2 Twelve lead ECG during catheter ablation for right ventricular outflow tract premature ventricular complexes (PVC). The coupling interval of the PVC couplet became longer after the onset of radiofrequency application $(+720 \mathrm{~ms})$ than before $\left({ }^{\star} 260 \mathrm{~ms}\right)$. Five seconds after radiofrequency energy began to be delivered, PVCs disappeared completely.

administration of lignocaine (lidocaine) $100 \mathrm{mg}$, procainamide $1000 \mathrm{mg}$, adenosine triphosphate (ATP) $20 \mathrm{mg}$, and verapamil $5 \mathrm{mg}$ was ineffective at suppressing these premature ventricular complexes. As the ventricular fibrillation was refractory to drug treatment and was always triggered by a premature ventricular complex from the same focus, we decided to eradicate the culprit complexes by radiofrequency catheter ablation, targeting the focus in the right ventricular outflow tract.

The ablation site was determined by activation mapping, using a 64 pole basket catheter, and by pace mapping using a $4 \mathrm{~mm}$ tip ablation catheter. During bigeminal premature ventricular complexes, the earliest activation potential was recorded at the posteroseptal right ventricular outflow tract $30 \mathrm{~ms}$ before QRS onset in the isolated premature ventricular complex. The QRS morphology during pacing from that site showed a $12 / 12$ match with the spontaneously occurring premature ventricular complexes. With the temperature control set at $55^{\circ} \mathrm{C}$, radiofrequency energy was delivered for 120 seconds. Premature ventricular complexes almost disappeared within five seconds of the application of radiofrequency energy (fig 2). Additional ablation was applied three times around this site, and ventricular fibrillation was no longer inducible even with triple extrastimuli from the right ventricle. A Holter ECG recorded five days after the ablation showed that the number of premature ventricular complexes had been reduced to 310 a day. The patient was discharged on no antiarrhythmic drugs. Over the subsequent 20 months, no episode of ventricular fibrillation or shock delivery from the ICD has been noted.

\section{Discussion}

Idiopathic ventricular fibrillation is regarded as an entity in which there is ventricular fibrillation of undetermined aetiology in the absence of structural heart disease or any reversible or transient cause. ${ }^{1}$ The therapeutic approach to this condition is limited, and only an ICD can save life. ${ }^{2}$ However, although the ICD is a powerful device for terminating ventricular fibrillation, it cannot prevent its initiation and the ensuing syncope. Antiarrhythmic drugs are often prescribed to prevent recurrences of ventricular fibrillation, but their efficacy remains to be established. ${ }^{3}$ Our report describes a case of idiopathic ventricular fibrillation causing a repetitive ICD discharge which was successfully treated by eradicating the premature ventricular complex responsible for initiating the ventricular fibrillation, using radiofrequency catheter ablation.

These are several varieties of idiopathic ventricular fibrillation. Brugada and Brugada reported a syndrome characterised by a right bundle block pattern and ST elevation in the right precordial leads. ${ }^{5}$ This diagnosis was unlikely in our case as there was no ST elevation in the right precordial leads, even immediately after the episode of ventricular fibrillation. Leenhardt and colleagues described a short coupled variant of torsade de pointes as a variant of idiopathic ventricular fibrillation. ${ }^{6}$ In their report, polymorphic ventricular tachycardia was induced by a short coupled premature ventricular complex which had a coupling interval of $245 \mathrm{~ms}$, and the origin of the premature ventricular complex was either in the right or the left ventricle. In contrast to their report, ventricular fibrillation in our case was triggered by a complex with a longer coupling interval of 320-330 ms, which was the same as the coupling interval in premature ventricular complexes that did not provoke ventricular fibrillation.

A beneficial role of radiofrequency catheter ablation for symptomatic premature ventricular complexes has been reported. ${ }^{7}$ Our case is unique in that catheter ablation targeting a monofocal complex suppressed the induction of ventricular fibrillation. It is, however, uncertain whether this intervention only eliminated a trigger for ventricular fibrillation or whether it ablated a common arrhythmogenic site responsible for both premature ventricular complexes and ventricular fibrillation. Had the ablation treated only a trigger, it is possible that the substrate for ventricular fibrillation is still present and ICD will remain necessary for this patient. Although our inability to induce ventricular fibrillation by programmed stimulation suggests a modification of the fibrillation substrate by the radiofrequency energy, this does not exclude the possibility that the substrate for ventricular fibrillation persists, though surrounded by protective areas.

\section{CONCLUSIONS}

A case of idiopathic ventricular fibrillation was successfully treated by radiofrequency catheter ablation. When the development of idiopathic ventricular fibrillation is reproducibly triggered by premature ventricular complexes of monofocal origin, catheter ablation targeting this culprit premature ventricular complex may be an option for preventing the recurrence of ventricular fibrillation. 
1 Viskin S, Belhassen B. Idiopathic ventricular fibrillation. $A m$ Heart F 1990;120:661-71.

2 Mital S, Iwais, Stein Kis at al. Long-term outcome of patients with unexplained syncope treated with an electrophysiologic-guided approach in the implantable cardioverter-defibrillator era. F Am Coll Cardiol 1999;34: 1082-9.

3 Ruskin JN. Idiopathic ventricular fibrillation: is there a role for electophysiologic-guided antiarrhythmic drug therapy? f Cardiovasc Electrophysiol 1999;10:1313-15.

4 Poole JE, Mathisen TL, Kudenchuk PJ, et al. Long-term outcome in patients who survive out of hospital ventricular
fibrillation and undergo electrophysiologic studies: evalua- tion by electrophysiologic subgroups. I Am Coll Cardiol 1990;16:657-65.

5 Brugada P, Brugada J Right bundle branch block, persistent ST segment elevation and sudden cardiac death: a distinct clinical and electrographic syndrome. A multicenter report. f Am Coll Cardiol 1992;20:1391-6.

6 Leenhardt A, Glaser E, Burguera M, et al. Short-coupled variant of torsade de pointes. A new electrocardiographic entity in the spectrum of idiopathic ventricular tachyarrhythmias. Circulation 1994;89:206-15.

7 Lauck G, Burkhardt D, Manz M. Radiofrequency catheter ing in the right outflow tract. PACE 1999;22:5-16. 